\title{
Arte y Salud:
}

\section{algunas reflexiones para profundizar las potencialidades de análisis del campo}

Gabriela Wald(a)

Wald G. Arts and Health: some thoughts for deepening the potential of analysis in this field. Interface (Botucatu). 2015; 19(55):1051-62.

This paper discusses the most common principles of the "arts and health" field, and specifically studies from Anglo-Saxon countries that analyze community-based art programs. It presents results from a qualitative study that was conducted within two youth orchestra projects in the city of Buenos Aires, Argentina, which had the aim of social integration. This study showed how the possibilities for an art education project to have an influence depend not only on what the programs can offer, but also fundamentally on who the participants are and can bring along: their universe of beliefs and standards, interpretation schemes, moral stances, cultural repertoires, practical knowledge, memories and economic, occupational and educational paths. These factors, which stem from prior socialization processes, constitute a form of filter or sieve through and from which artistic interventions may or may not have appropriate results.

Keywords: Youth orchestras. Arts and health. Young people. Vulnerability. Artistic education.
El articulo discute las asunciones más corrientes del campo "arte y salud", específicamente aquellos estudios del mundo anglosajón que analizan los programas de arte basados en la comunidad. Presenta resultados de una investigación cualitativa realizada en dos proyectos de orquestas juveniles con fines de integración social de la Ciudad de Buenos Aires, Argentina. El trabajo muestra cómo las posibilidades de influencia de un proyecto de educación artística dependen no sólo de lo que los programas ofrecen sino, fundamentalmente, de aquello que los participantes traen y son: sus universos de creencias y normativos, sus esquemas de interpretación, posicionamientos morales, repertorios culturales, saberes prácticos, memorias, trayectorias económicas, laborales, educativas. Estos aspectos, que son resultado de procesos de socialización previos, constituyen una suerte de filtro o tamiz a través y a partir de los cuales las intervenciones artísticas pueden - o no - ser apropiadas.

Palabras clave: Orquestas juveniles. Arte y Salud. Jóvenes. Vulnerabilidad. Educación artística.

\footnotetext{
(a) Conicet. Universidad de Buenos Aires, Instituto de Investigaciones Gino Germani, Área de Salud y Población. Uriburu 950, 6to piso of. 3, 1114, Buenos Aires, Argentina. gawald@gmail.com
} 


\section{Introducción}

El presente trabajo aporta al debate de un reciente campo de intervención y estudio denominado "arte y salud", tomando como referencia la perspectiva desarrollada en el mundo anglosajón $n^{1,2}$.

Las relaciones entre arte y salud se han discutido durante los últimos 30 años en diversas latitudes incluyendo América latina ${ }^{3-6}$. En Gran Bretaña a partir de 1990 el campo tuvo un gran desarrollo $0^{1,2,5,7}$, como consecuencia de la implementación de un abanico de intervenciones novedosas basadas en una concepción amplia e integral de salud. Antes de eso, las intervenciones en arte y salud solían confinarse a hospitales o clínicas, o al uso del teatro, la música, la pintura y otras artes para la comunicación de mensajes saludables ${ }^{1,8}$.

A partir del surgimiento de esas intervenciones, distintos académicos - nos centraremos aquí principalmente en los académicos anglosajones - comenzaron a investigar de manera sistemática cómo las artes impactaban en el bienestar de las poblaciones, incluyendo el análisis de actividades en ámbitos sanitarios y fuera de ellos, en las comunidades ${ }^{1,2,7-9}$.

El contexto socio-político para el nacimiento de este campo lo constituyeron las nuevas agendas transversales en política social de occidente, basadas en la idea del Desarrollo Humano ${ }^{10}$ y preocupadas por temáticas como la cohesión comunitaria, la inclusión social y el desarrollo sustentable ${ }^{2,11}$. En paralelo, la esfera cultural cobró en los años 1990 un protagonismo mayor que en momentos anteriores de la historia, cuestión que se evidenció en el uso creciente de todo tipo de prácticas artístico-culturales como herramientas para el mejoramiento sociopolítico y económico ${ }^{12}$.

En términos teóricos, el campo del arte y la salud es heredero del concepto positivo e integral de salud - entendido no como ausencia de enfermedad sino como un proceso de bienestar colectivo influenciado por factores económicos, psico-sociales, culturales y ambientales ${ }^{13-15}$. Asimismo, influyeron las teorías del capital social, que sugerían que la participación en proyectos locales podía mejorar la comunicación y las relaciones comunitarias ${ }^{16,17}$, a la vez que incrementar la sensación de bienestar y autoestima ${ }^{18}$.

El uso del arte para promover objetivos sociales no es una idea nueva. Durante los años 1960 diversas iniciativas para trabajar con poblaciones excluidas fueron ofrecidas por artistas y organizaciones vinculadas a las artes ${ }^{19}$. Lo que sucede a partir de la década de 1990 es que iniciativas de arte en la comunidad comienzan a ser implementadas desde el sector salud - en conjunto con otros sectores - en un ejercicio interdisciplinario que convoca actores tanto de campo de la cultura y las artes como de desarrollo social, educación artística, psicología social y comunitaria, entre otros 1,9,20.

Como consecuencia de la extensión de proyectos de arte que buscan promover el bienestar, la investigación en el marco de los mismos dio lugar a un conjunto creciente de trabajos que estudian los posibles beneficios del arte sobre la salud integral.

Para sintetizar la diversidad de enfoques y actividades que se incluyen en el campo, Macnaughton, White y Stacy ${ }^{8}$ realizaron el diagrama de la Figura 1 - basado en uno desarrollado por Smith 9 .

Como puede observarse, el campo es heterogéneo. Incluye iniciativas focalizadas en los beneficios terapéuticos de las artes así como otras que buscan establecer mejoras en los servicios de salud, desarrollar campañas participativas en salud, y aquellas con objetivos transversales como mejorar el capital social y los vínculos comunitarios, o generar espacios de integración social.

Como consecuencia de esta diversidad, los estudios del campo han generado literatura con dos objetivos principales: establecer conexiones teóricas entre arte y salud - en particular para aquellas intervenciones comunitarias - y proveer evidencia empírica de dichas conexiones.

En el presente trabajo me propongo discutir algunas de las asunciones más corrientes que circulan en este campo, utilizando resultados de una investigación cualitativa realizada en el marco de dos proyectos de orquestas juveniles en dos barrios populares de la Ciudad de Buenos Aires, Argentina. Considero que los resultados que aquí se presentan pueden aportar elementos sustantivos para la discusión de los vínculos entre intervenciones artísticas y cambios en los participantes fundamentalmente por las preguntas problema y el enfoque epistemológico propuesto. 
'La cohesión social es salud'

Proyectos que enfatizan la creatividad como herramienta para mejorar las relaciones comunitarias. Reflejan una corriente de pensamiento en expansión que afirma que las buenas relaciones son un determinante importante de la salud.

\section{Comunidad}

\section{Comprometer grupos}

Proyectos que se proponen comprometer a distintos actores sociales para acercar el arte a la promoción de la salud. Utilizan métodos creativos para explorar, diseminar y comunicar mensajes vinculados con la salud.
Creatividad y bienestar Proyectos que enfatizan la creatividad como camino hacia el bienestar. Utilizan abordajes creativos para favorecer la expresión personal. El arte es entendido como una suerte de terapia

\section{Individuos}

Apoyo a los servicios de salud Proyectos que apoyan el cuidado de personas con problemas de salud utilizando el arte y la creatividad. Arte por prescripción. Ámbitos de cuidado.

Servicios de Salud

Figura 1. Dimensiones centrales en Arte y Salud

Fuente: Macnaughton, White y Stacy ${ }^{8}$.

Sitúo el debate en el cuadrante superior izquierdo del diamante arriba presentado, dialogando con aquellos trabajos que analizan intervenciones de arte en la comunidad sin contenidos específicos de salud. Estas intervenciones de carácter colectivo con foco en el arte pueden ser analizadas por una gran variedad de disciplinas y campos de trabajo, entre los cuales se cuentan la educación artística, la educación comunitaria, la educación no formal, las políticas sociales, las políticas culturales con sectores populares, la educación popular, la psicología social y comunitaria, la antropología, la sociología y hasta la economía. Si bien los resultados que aquí se presentan pueden resultar útiles y pertinentes a todos esos campos, es en la arena sanitaria donde aún predominan modelos explicativos naturalistas y causalistas que casi nunca conviven con la complejidad que requiere el análisis de las intervenciones sociales en contextos de vulnerabilidad social, en particular cuando involucran elementos de la cultura y las artes. Por eso el interés que este trabajo tiene para dicho campo.

\section{Las conexiones entre arte y salud: la búsqueda de indicadores intermedios}

Para comprender por qué las intervenciones de arte en la comunidad son pertinentes al campo de la salud es central comprender el concepto positivo e integral de salud ${ }^{20}$. Si bien el mismo no ha estado exento de críticas y contradicciones - si todo es dominio de la salud entonces nada lo es, dicen sus principales detractores - el mundo occidental lo ha adoptado como propio hace ya varias décadas. Reconoce como pertenecientes a su campo problemático las prácticas, situaciones o condiciones capaces de producir tanto bienestar como malestar en los sujetos, grupos y comunidades ${ }^{15}$.

En líneas generales, los diferentes estudios llevados a cabo en intervenciones comunitarias de arte y salud afirman que estas experiencias son beneficiosas para la salud y el bienestar de sus participantes. Las mismas pueden promover sensaciones de bienestar, emociones positivas, auto confianza, auto valoración, capacidades para la socialización, relaciones grupales positivas, redes comunitarias, disfrute, diversión, distensión, cohesión, compromiso, comunicación comunitaria, reducción del estrés, etc.1,2,8,21-29.

Dada la prevalencia dentro del sector salud de la investigación cuantitativa, y la dificultad existente en el mismo para incorporar o al menos dialogar con paradigmas de investigación interpretativos, los estudios en arte y salud se han centrado en demostrar los beneficios del arte en la mejora de la vida de 
las personas a partir de la definición de indicadores intermedios de salud individual y colectiva. Es decir, aplicaron indicadores blandos dentro de un modelo de abordaje inspirado en las ciencias duras.

Los estudios analizan dos grandes grupos de dimensiones e indicadores vinculados a proyectos de arte comunitario. El primer grupo está compuesto por los resultados en términos de salud amplia y bienestar que las iniciativas artísticas pueden lograr, mientras que el segundo aborda los procesos pedagógicos y las metodologías de intervención que facilitarían o dificultarían esos resultados.

Dentro de los resultados que los proyectos pueden lograr, los investigadores identifican factores individuales (autovaloración, autoestima, diversión, confianza en uno mismo, orgullo por los logros conseguidos, sensaciones de disfrute, bienestar y pertenencia, reducción del estrés, habilidades expresivas, motivación, compromiso, comprensión de uno mismo, habilidades para la negociación y resolución de problemas, desarrollo de identidades positivas) y factores colectivos: al interior del grupo (amistad, sociabilidad, relaciones de confianza, habilidades para la organización) y con la sociedad (construir una imagen positiva de las personas que viven en contextos de vulnerabilidad social, erigir capacidades locales basadas en aspectos comunitarios positivos, combatir inequidades en salud, generar orgullo de los grupos marginados, incremento del sentido del derecho, de redes sociales, de vínculos comunitarios e inclusión social) 1,2,5,8,9,11,22,21,23-27,29,30-32.

En relación a los procesos que facilitan dichos resultados, la literatura sugiere que son más exitosos aquellos proyectos que a) focalizan en los procesos creativos e imaginativos, y en la producción de arte de calidad; b) no necesariamente establecen vínculos ente las actividades artísticas y contenidos sociales o educacionales (muchos participantes desaprueban estas conexiones); c) construyen espacios distendidos y acogedores que facilitan la interacción entre los participantes y de éstos con los docentes; y d) trabajan para que la comunidad local se involucre y apropie del espacio que se ha generado $24-28$.

Asimismo, los estudios señalan que iniciativas de arte comunitario pueden producir cambios en dimensiones colectivas de salud en las cuales las intervenciones de base biomédicas no han sido efectivas ${ }^{20,32}$ y por lo tanto, pueden ser consideradas un medio efectivo de promoción de la salud. Sin embargo, la potencialidad de cada proyecto depende de su modelo de intervención, de la estrategia pedagógica, de los modelos de participación que promueve y hasta de ciertos individuos ${ }^{25,26}$.

A simple vista lo expuesto en este apartado pareciera ser un modelo disponible para el análisis de experiencias de arte comunitario, aplicable a contextos y situaciones diversas. El mismo permitiría establecer vínculos - o afirmar que no existen - entre estas experiencias y aspectos de salud integral vía la presencia o no de indicadores intermedios. Ahora bien, ¿qué lugar otorgan estos estudios al bagaje que cada persona trae consigo cuando se acerca a un proyecto de arte comunitario? ¿Es posible pensar en los "efectos" de un programa de arte comunitario focalizando sólo en aquello que las iniciativas ofrecen? ¿Qué lugar tiene en este tipo de modelos explicativos el interjuego entre condiciones estructurales, repertorios culturales y trayectorias biográficas de los participantes - cuestiones que al desplegarse van configurando apropiaciones diferenciales de los proyectos? Si la estética y el arte son nociones/prácticas socialmente construidas y situadas ¿por qué se las analiza sin hacer referencia a estas cuestiones?

En el apartado siguiente se describirán los dos proyectos de orquestas juveniles en los cuales se realizó la investigación que se presenta, así como la metodología utilizada. En los apartados que le siguen se analizarán algunos resultados, en diálogo con ciertas ideas del campo del arte y la salud.

\section{Apuntes sobre los casos y la metodología}

Como mencioné anteriormente, este artículo presenta algunos resultados de una investigación realizada en el marco de dos proyectos de orquestas juveniles en dos barrios populares de la Ciudad de Buenos Aires.

Los principales objetivos fueron a) comprender los sentidos que los jóvenes y sus padres/madres otorgaban al paso por los proyectos de orquestas juveniles, b) analizar los modos en que se apropiaban de aquello que los proyectos les proponían y c) indagar qué elementos podrían estar influyendo en 
(b) Para resguardar el anonimato y la confidencialidad de las y los entrevistadas/os se optó por no revelar los nombres de las orquestas estos sentidos y apropiaciones. Si bien estos procesos parecieran distanciarse bastante de lo que proponen los estudios en "arte y salud", tanto los "sentidos" de las orquestas como sus "usos" o "apropiaciones" implican indagar en las categorías e indicadores que dicho campo propone, pero desde una perspectiva que totalice la experiencia vivida. Así, el análisis aquí propuesto avanza sobre procesos complejos que pueden o no dar lugar a los indicadores intermedios enunciados más arriba (individuales como autoestima, confianza en uno mismo, disfrute, etc. y colectivos, como sociabilidad, habilidades para la organización, construir una imagen positiva de los sectores populares, etc.) pero desde una postura teórica y epistemológica que complejiza las lecturas causalistas que priman en el campo del arte y la salud.

Me propuse asimismo indagar si existían regularidades entre los sentidos y usos de las orquestas y distintas características de los jóvenes y sus familias (posición socioeconómica, trayectorias educativa, laboral, residencial; y repertorios culturales).

Para llevar adelante la investigación opté por un enfoque cualitativo, interpretativo y de inspiración etnográfica pues las dos características básicas del método etnográfico 1) la presencia sostenida del investigador en el terreno donde tienen lugar las prácticas que analiza y 2) el trabajo con los sentidos y representaciones de los actores a partir de sus puntos de vista ${ }^{33,34}$ se revelaron centrales para abordar las preguntas problema de este estudio.

La investigación constó de dos etapas. La primera comenzó en abril del año 2010 e implicó la inserción la que llamaremos la Orquesta Juvenil $A^{(b)}$ durante un año y medio. La segunda comenzó en agosto de 2010 con la inserción en la que llamaremos la Orquesta Juvenil B y se extendió hasta marzo de 2012. Ambos proyectos se desarrollaban en dos barrios del sur de la Ciudad.

Los dos proyectos seleccionados resultaban a grandes rasgos similares: se proponían acercar la música académica y la práctica orquestal a sectores sociales habitualmente alejados de ellas ofreciendo clases de instrumentos, de lenguaje musical y práctica en orquesta. Entregaban un instrumento en comodato a cada uno de los participantes, para que todos tuvieran la oportunidad de practicar en sus casas. La dirección artística de ambos proyectos estaba a cargo de la misma persona (un reconocido músico y director de un conservatorio de la Ciudad) y por tal razón, los repertorios eran similares (variaba la dificultad de acuerdo a las posibilidades de interpretación de cada joven).

Ambos proyectos presentaban también algunas diferencias. La Orquesta $A$ estaba gestionada por el área del cultura del Gobierno de la Ciudad de Buenos Aires mientras que la Orquesta B por el área de educación. En la Orquesta A la mayor parte de los jóvenes vivía en una villa de emergencia cercana (favela), mientras que en la Orquesta B la composición socio-económica era más heterogénea: jóvenes provenientes de viviendas sociales, de villas de emergencia, de barrios populares cercanos y de las zonas residenciales del barrio, estas últimas habitadas por clases medias. Asimismo, en la Orquesta $A$ los jóvenes comenzaban a participar a partir de los 13 años, mientras que en la Orquesta B comenzaban de niños, entre los 6 y los 12 años.

El abordaje metodológico de la investigación implicó la triangulación de información obtenida a lo largo de un año de observaciones (con y sin participación) en cada orquesta, 8 grupos de discusión con jóvenes, 42 entrevistas en profundidad ( 22 a jóvenes participantes, 12 a padres y/o madres y 8 a docentes $u$ otros gestores), observaciones esporádicas posteriores a las entrevistas, análisis de documentos producidos por los proyectos y lectura de notas periodísticas. 
Se relevó información que pudiera dar cuenta de los sentidos que cada uno de los actores otorgaba a los proyectos, a la práctica orquestal, a la interpretación musical y si identificaban algún tipo de transformación en los participantes. Asimismo, se relevó información sobre datos de posición socioeconómica, trayectorias (educativas, laborales, residenciales, familiares) y repertorios culturales de los jóvenes y sus familias, y sobre los modos en que se perciben a sí mismos en relación a sus docentes y otros gestores pero también en relación a sus pares, parientes, vecinos y amigos.

\section{La investigación en las orquestas juveniles: principales resultados}

El estudio llevado a cabo muestra que si bien aparecieron diferencias en algunos aspectos entre ambos proyectos de orquestas juveniles, los hallazgos más significativos resultaron transversales a ambas iniciativas. Estos resultados avanzan sobre cuestiones que son de interés para diversos campos de estudio, no obstante, los estudios en arte y salud en Europa y Gran Bretaña se han focalizado en el análisis de intervenciones artísticas comunitarias desde una perspectiva que disecciona, operacionaliza y fracciona procesos con el fin de dialogar con los paradigmas de investigación biomédicos. Deviene importante complejizar los principales argumentos de dicho campo, a partir del análisis de algunos aspectos que median entre la implementación de los programas y sus posibles efectos.

Expondré a continuación algunas reflexiones derivadas del análisis de las trayectorias educativas, laborales, familiares y residenciales de las familias de los jóvenes participantes de las dos orquestas juveniles, así como de las adscripciones morales a las cuales manifestaban adherir estas familias. Pues los primeros resultados de mi investigación confirmaban uno de sus supuestos iniciales: que las posibilidades de influencia de un proyecto de educación artística entre sus participantes no dependían sólo de lo que los programas ofrecían sino que adquiría un lugar fundamental aquello que los jóvenes traían consigo como resultado de procesos de socialización previos, todo lo cual constituía una suerte de flitro o tamiz a través y a partir del cual los programas de orquestas podían ser apropiados. La idea de que existe algo entre el programa y sus "efectos" no es para nada revolucionaria. Sin embargo, los estudios sobre intervenciones socio-comunitarias suelen dejarla de lado, evaluando fundamentalmente la correlación entre objetivos y resultados de programas.

En las dos orquestas juveniles que se analizaron aparecieron recorridos heterogéneos entre los padres de los jóvenes en aspectos laborales, educativos, residenciales y familiares. No obstante, surgieron algunas características que compartían prácticamente todas las familias entrevistadas, cuestión que convocó mi atención. En primer lugar, todas contaban con al menos uno de sus integrantes empleado en condiciones formales -algunos de ellos manteniendo sus empleos por diez o quince años. Vale aclarar aquí que en la Argentina esta situación es excepcional entre familias de barrios populares o medio-bajos, en los cuales viven casi todos los integrantes de ambos proyectos orquestales. En estos barrios sólo el 15\% de los jefes/as de hogar poseían empleos registrados al momento del trabajo de campo ${ }^{35}$. Así, la condición laboral colocaba a las familias de los jóvenes de ambas orquestas en un lugar diferencial del de la mayoría de sus vecinos, vinculados al mundo del trabajo informal o inestable, de la asistencia, el desempleo o combinaciones de estas cuatro variantes ${ }^{35}$.

En segundo lugar, hallé que las familias de los jóvenes de ambas orquestas compartían un conjunto de posicionamientos que referían a criterios de orientación moral, expresados en valores como la perseverancia, el esfuerzo, la responsabilidad, la honestidad y el sacrificio. Estos valores no eran sólo pregonados de manera retórica sino que muchas de sus acciones parecían orientadas por ellos. Las referencias a dichas orientaciones valorativas aparecían en sus relatos sobre trayectorias residenciales, laborales, afectivo-familiares y en algunas cuestiones que demandaban de sus hijos, como ser la finalización de sus estudios secundarios, la consecución de estudios terciarios/universitarios o la búsqueda de un trabajo en lo posible formal. Como consecuencia de su socialización en estos repertorios morales, casi la totalidad de jóvenes que participaron del estudio en ambas orquestas desafiaban las trayectorias típicas que los estudios sociológicos describen como prevalentes en jóvenes de sectores empobrecidos y populares en la Argentina actual ${ }^{35-44}$ : en lugar de abandonar el secundario lo terminaban (más de la mitad sin repetir ningún año), en lugar de tener hijos a edades tempranas 
retrasaban los proyectos de maternidad o paternidad con el objetivo de conseguir un trabajo o iniciar una "carrera" laboral. Muchos incluso iniciaban estudios terciarios o universitarios.

\section{Ahora bien, ¿qué nos dicen estos hallazgos?}

En líneas generales, pareciera que en ambas orquestas convergen cierto tipo de familias de barrios populares y no otras. Es decir, que no cualquier joven de estos barrios puede acercarse y permanecer en estas iniciativas. Los que lo hacen pertenecen a familias que, a diferencia de lo que plantean diversos estudios sociológicos recientes en poblaciones empobrecidas o de bajos recursos en la Argentina ${ }^{36,37,42}$, todavía orientan sus estrategias de reproducción en base a valores meritocráticos (perseverancia, esfuerzo, responsabilidad, honestidad, sacrificio). Estos valores solían guiar las prácticas educativas y laborales de las clases medias y trabajadoras argentinas durante el siglo $X X^{45}$. Pero, según la sociología reciente, la meritocracia habría desaparecido en los sectores populares y medio bajos, como consecuencia de profundos cambios estructurales que generaron procesos de fragmentación y exclusión social. Las nuevas estrategias de reproducción de los sectores populares se basarían más bien en una lógica que privilegia lo inmediato y posible frente a lo que lleva tiempo y no es seguro de poder conseguir (por ejemplo un diploma de escuela secundaria o un empleo formal), y el corto plazo frente a la planificación ${ }^{38,39,43,44}$.

El dato sobre los repertorios morales de las familias de los jóvenes debe leerse en conjunto con otros derivados del tipo de propuesta que ofrecen las orquestas. En primer lugar, ambas iniciativas implican constancia, alta frecuencia semanal de clases y ensayos (entre dos y tres veces a la semana), interfieren horarios que pueden alterar las rutinas familiares (funcionan en horario vespertino y una de ellas también sábados), suponen la disponibilidad de los participantes para asistir a conciertos y requieren resolver el traslado hacia las sedes donde funcionan. Todo lo cual supone una intensa demanda a las familias, quienes se encuentran ya exigidas por la superposición de tareas y actividades de producción material y reproducción familiar.

En segundo lugar, debe tenerse en cuenta que las orquestas también suponen y promueven orientaciones valorativas. Me permito citar aquí las palabras de un docente:

"La orquesta es como una pequeña sociedad. Cada uno tiene que aprender a cumplir un rol, escucharse entre ellos, escuchar al director, y todos tienen que caminar juntos [...] En una orquesta estás ocupando un espacio, si aprendiste y estudiaste vas progresando, en vez de estar en el último atril vas a estar en el primero [...] Estudiar música implica poner monedas en una alcancía [...] Es una habilidad acumulativa que empieza a fomentar algunos valores: la perseverancia, la voluntad de sentarse a hacer algo con continuidad durante mucho tiempo. Son una serie de valores difíciles para los tiempos que corren [...] Hay que comprar hoy y mañana tirar. Estudiar no vale la pena, hay que copiarse. Nosotros, casi sin darnos cuenta, estamos fomentando otros valores". (Docente Orquesta B)

Es sencillo observar que los valores que según este docente son promovidos por el proyecto orquestal se corresponden con aquellos identificados en los discursos de los padres de los jóvenes como aquellos que guían sus acciones y decisiones.

Por lo expuesto hasta aquí, pareciera existir una afinidad electiva ${ }^{46}$ entre las prácticas promovidas por los proyectos, y los repertorios culturales y morales en los que han sido socializados los jóvenes. Así, la permanencia de los jóvenes en las orquestas estaría dependiendo no sólo del gusto por aquello que los programas les ofrecen sino, fundamentalmente, de que ellos y sus familias compartan con los proyectos ciertos rasgos culturales y morales que les permitan valorar y priorizar estos espacios. Pues si las familias priorizan la actividad de las orquestas, logran responder a las exigentes demandas que implican los proyectos para ellas. Asimismo, si los niños y jóvenes que se inician en las orquestas no comulgan con el esfuerzo, el sacrificio y el largo plazo que las mismas implican, tampoco logran permanecer en ellas. En Argentina, por lo que se ha dicho, no es evidente que una familia en 
condiciones de vulnerabilidad social priorice una actividad de este tipo para sus hijos.

Ahora bien, cuando esto ocurre, cuando se alinean algunos de los repertorios culturales y morales entre las familias, los jóvenes participantes y los proyectos, es posible que los mismos puedan producir resultados poco frecuentes en barrios populares: jóvenes que se profesionalizan en la música académica, que terminan la escuela secundaria y eligen estudiar en la universidad o realizar estudios terciarios ${ }^{(c)}$, que adoptan la idea de vocación o carrera profesional, que aman la música y sus instrumentos, que encuentran en las orquestas un espacio de socialización fundamental y distinto de todos los que existen en sus barrios, que se sienten orgullosos de lo que han logrado, que creen en ellos mismos y en lo que pueden hacer, que disfrutan de tocar frente a distintos públicos, que se sienten valorados por personas de otros sectores sociales, que circulan y conocen distintas zonas de la ciudad, y que arman un proyecto biográfico muy diferente al que, según ellos, hubieran armado de no haber mediado las estos proyectos.

Así, cuando todo esto que ocurre, es posible identificar los indicadores intermedios que describen los estudios del campo del arte y la salud:

autovaloración, autoconfianza, autoestima, sensaciones de disfrute, de bienestar, de pertenencia, habilidades expresivas, capacidades para la socialización, relaciones grupales positivas, motivación, compromiso, orgullo por los logros conseguidos, etc.

No obstante, los hallazgos sugieren que es ingenuo pensar que todo niño/a, adolescente o joven que así lo desee puede acercarse y permanecer en proyectos artísticos como los aquí analizados - en particular de sectores populares, donde la organización hogareña raras veces puede convivir con las demandas y exigencias que requieren estas iniciativas.

La investigación realizada - que aquí se presenta sólo en parte - permite afirmar que las posibles influencias de las orquestas en las trayectorias biográficas de los jóvenes son el resultado específico de intersecciones también específicas entre 1) los mandatos de una política o intervención artístico-cultural, 2) lo particular que aportan quienes la llevan adelante (gestores), 3) la contingencia del encuentro entre gestores y participantes, en un tiempo y un barrio determinados, y 4) las trayectorias biográficas y repertorios culturales que ponen en juego los "beneficiarios". Estas dimensiones no son estáticas sino procesos dinámicos que a la vez se influencian mutuamente, y que ocurren en el marco de procesos estructurales también cambiantes que van configurando, entre otras cosas, los modos de pensar la política social y cultural en el mundo occidental.

Esto no quiere decir que proyectos de este tipo no deban realizarse; más bien todo lo contrario: la propuesta es que se lleven a cabo con total conocimiento de sus límites (el hecho de que no tendrán el mismo impacto en todos los casos) y de sus múltiples potencialidades, que son de gran importancia para las poblaciones que buscamos integrar con la programática social.

\section{Algunas reflexiones finales}

Los resultados presentados hasta aquí son pertinentes, como hemos dicho, a una variedad de campos disciplinares vinculados a las intervenciones comunitarias - de los cuales el del arte y la salud es sólo uno de los posibles. Si (c) En Argentina el porcentaje de jefes/ as de hogar de barrios populares (urbanizaciones informales +

urbanizaciones formales de nivel socioeconómico bajo) que terminan el secundario es del $22 \%$ (ODSA, 2011) y el porcentaje que ingresa a la universidad es ínfimo). 
bien las orquestas juveniles analizadas no fueron pensadas como iniciativas para promover la salud, la participación en las mismas sí puede impactar sobre los llamados "indicadores intermedios de la salud" desarrollados en los estudios citados. Puesto que en este campo continúan siendo hegemónicos los modelos explicativos derivados de las ciencias naturales, considero importante discutir con él y proponer la introducción de un paradigma interpretativo.

Así, a partir de lo expuesto hasta aquí, ¿qué ideas puede aportar la investigación realizada en las dos orquestas juveniles a la programática social comunitaria? ¿Y al campo del arte y la salud?

En primer lugar, que para comprender en profundidad el tipo de "impacto" de una intervención socio-cultural en sectores populares es preciso superar ciertos esquematismos teórico-metodológicos y plantear formas de investigar que puedan registrar con la mayor complejidad posible los universos simbólicos y normativos de los "destinatarios" de las políticas, así como los recorridos que los han llevado hasta allí. Aunque ya se ha dicho muchas veces, gran parte de la investigación social no logra aún dejar de proyectar sus modos de entender y concebir el mundo en las interpretaciones que realizan de sus "objetos" de estudio. En el caso de estudios sobre intervenciones sociales esto incluye también complementar los estudios desde arriba, es decir, aquellos que hacen foco en las potencialidades de ciertas propuestas o programas, con estudios desde abajo, es decir, atendiendo a los modos en que las intervenciones pueden ser apropiadas (y de qué modos) por sus participantes.

En segundo lugar, es preciso comprender la heterogeneidad de actores, modos de pensar, hacer y trayectorias que se agrupan bajo los calificativos o conceptos con que habitualmente se piensan los grupos y sectores (clases) sociales. En el caso que nos ocupa es necesario tener en cuenta la heterogeneidad y diversidad existente dentro de lo que llamamos "sectores populares" o "mediobajos" y evitar suponer que lo que se ofrece será accesible, interesante o "bueno" para todos - o para las mayorías. Pues lo que cada intervención comunitaria pueda o no lograr dependerá tanto de los individuos que la llevan adelante ${ }^{25}$ como de aquello - contingente, único e imposible de planificar - que sucede en el encuentro entre unos gestores y unos "destinatarios" de carne y hueso. Así, en el caso de realizar estudios en iniciativas socio-comunitarias (y esto excede el campo del arte y la cultura) se deben incorporar al análisis tanto aspectos referidos a las particularidades de los "beneficiarios" y sus recorridos previos como a aquello irrepetible que sucede cuando una política se encuentra con aquellos a quienes ha logrado convocar.

En tercer lugar, el estudio realizado en los dos proyectos de orquestas permite sugerir la idea de que toda intervención social - y en particular aquellas que se basan en una actividad artísticocultural - lleva implícitos ciertos valores que los hacedores de políticas o bien no perciben o dan por sentados. Estos valores influencian - en mayor o menor medida - quiénes pueden ser los destinatarios y permanecer en dichas políticas, a la vez que están implícitos en las rutinas y tiempos que las intervenciones proponen/demandan, limitando o posibilitando accesos. Es preciso interrogar el uso acrítico del arte y la creatividad como recursos para el bienestar, pues dicho uso olvida - o simplemente no prioriza - que las mismas son nociones socialmente situadas, y que esto influencia el modo en que una intervención basada en las artes puede ser recibida, por ejemplo, por personas con trayectorias biográficas, repertorios culturales y posiciones estructurales disímiles.

Lo dicho hasta aquí alerta sobre la expectativa de linealidad con la que a menudo se piensan las intervenciones sociales - y para el caso específico de las iniciativas en arte y salud implica la necesidad de interrogar como principal metodología cualitativa la búsqueda y medición de indicadores intermedios. Es probable que el campo haya desarrollado tal marco teórico-metodológico para ser reconocido por los organismos que financian intervenciones en el campo de la salud. No obstante, considero que son precisos modelos de investigación complejos que permitan analizar no sólo cuáles son las características de las mejores prácticas sino también dar cuenta del interjuego entre condiciones estructurales, repertorios culturales y biografías de quienes participan en las mismas, en particular si involucran disciplinas artístico-culturales. Pues estos se interponen y generan nuevas mediaciones entre los "destinatarios" y las intervenciones que considero deberían comenzar a contemplarse. 


\section{Referencias}

1. Clift S. Guest Editorial. Health Educ. 2005; 105(5):328-30.

2. Dooris M. A qualitative review of Walsall arts into health partnership. Health Educ. 2005; 105(5):355-73.

3. Silva LFR, Cipullo MAT, Imbrizi JM, Liberman F. Oficinas de música e corpo como dispositivo na formação do profissional de saúde. Trab Educ Saude. 2014; 12(1):189-203.

4. García Cabrero B, Klein Kreisler I. La construcción de ambientes educativos para la convivencia pacífica: el modelo pedagógico del programa SaludARTE. Sinectica [Internet]. 2014; 42 [acesso 2015 Fev 17]. Disponível em: http://www.scielo.org.mx/scielo. php?script=sci_arttext\&pid=S1665-109X2014000100006\&lang=pt

5. Avila Valdes N. Un modelo de integración de arte y salud en España: el proyecto curArte I+D. Hacia Prom Salud. 2013; 18(1):120-37.

6. Couto ZFS, Cezar-Vaz MR, Svaldi JSD. A arte como processo tecnológico de compreensão e (re)significação do trabalho em saúde. Texto Contexto Enferm. 2009; 18(3):568-76.

7. Clift S, Camic PM, Chapman B, Clayton G, Daykin N, Eades G, et al. The state of arts and health in England. Arts Health Int J Res Policy Pract. 2009; 1 (1):6-35.

8. Macnaughton J, White M, Stacy R. Researching the benefits of arts in health. Health Educ. 2005; 105(5):332-9.

9. Smith T. Common knowledge: the tyne and wear health action zone's arts and health project - interim evaluation report [Internet]. Durham: University of Durham; 2001 [cited 2014 Aug 19]. Available from: https://www.dur.ac.uk/resources/cahhm/reports/CK\% 20 INT\% 20RPT\% 20-\%20final\% 20version1.pdf

10. Sen A. Desarrollo y libertad. 2a ed. Barcelona: Planeta; 2000.

11. Jermyn $\mathrm{H}$. The arts and social exclusion: a review prepared for the arts council of England [Internet]. London: Arts Council England; 2001 [cited 2014 Aug 8]. Available from: http://www.artscouncil.org.uk/publication archive/arts-and-social-exclusion-areview-prepared-for-the-arts-council-of-england/

12. Yudice G. El recurso de la cultura: usos de la cultura en la era global. Barcelona: Gedisa; 2002.

13. Organización Mundial de la Salud. Constitución. New York: OMS; 1946.

14. Labonte R. A community development approach to health promotion. Edinburgh: Health Education Board for Scotland/Research Unit in Health and Behavioural Change, University of Edinburgh; 1998.

15. Kornblit AL. La promoción de la salud entre los jóvenes. Acta Psiquiatr Psicol Am Lat. 2010; 56(3):217-26.

16. Coleman JC. Foundations of Social Theory. Cambridge: Harvard University Press; 1990.

17. Putnam R. Bowling alone: the collapse and revival of American community. Nueva York: Simon and Schuster; 2000.

18. Campbell C, Wood R, Kelly M. Social capital and health. Londres: Health Education Authority; 1999.

19. Hamilton C, Hinks S. Arts for health: still searching for the Holy Grail. J Epid Commun Health. 2003; 57(6):401-2. 
20. Angus J. A review of evaluation in community-based art for health activity in the UK [Internet]. London: Health Development Agency; 2002 [cited 2014 Aug 19]. Available from: https://www.dur.ac.uk/resources/cahhm/reports/CAHHM\%20for\% 20HDA\% 20 J\% 20Angus.pdf

21. Argyle $E$, Bolton $G$. Art in the community for potentially vulnerable mental health groups. Health Educ. 2005; 105(5):340-54.

22. White M, Robson M. The potent arts. J Public Mental Health. 2007; 6(4):12-3.

23. White M. A 'homecoming' for arts in mental health". Life Day. 2009; 13(3):37-40.

24. South J. Community arts for health: an evaluation of a district programme. Health Educ. 2006; 106(2):155-68.

25. Everitt A, Hamilton R. Arts, health and community: a study of five arts in community health projects [Internet]. Durham: CAHHM; 2003 [cited 2014 Aug 19]. Available from: https://www.dur.ac.uk/resources/cahhm/reports/Arts $\% 20 \mathrm{Health} \% 20 \% 26 \% 20$ Community.pdf

26. Health Development Agency [HDA]. Art for health: a review of good practice in community-based arts projects and initiatives which impact on health and wellbeing. Londres: HDA; 2000.

27. Swan P. Promoting social inclusion through community arts. Ment Health Soc Incl. 2013; 17(1):19-26.

28. Kirrmann K. CoolTan arts - enhancing well-being through the power of creativity. Ment Health Soc Incl. 2010; 14(2):12-6.

29. Secker J, Heydinrych K. Open arts: promoting well-being and social inclusion through art. Life Day. 2009; 13(4):20-4.

30. Grabowski D. Identity, knowledge and participation: health theatre for children. Health Educ. 2013; 113(1):64-79.

31. Spencer G. Young people's perspectives on health: empowerment, or risk? Health Educ. 2013; 113(2):115-31.

32. Carson A, Chappell N, Knight C. Promoting health and innovative health promotion practice through a community arts centre. Health Prom Pract. 2007; 8(4):366-74.

33. Wolcott $\mathrm{H}$. Some thoughts about ethnography. In: Anais do XIO Simposio Interamericano de Investigación Etnográfica en Educación; 2006; Buenos Aires, Argentina. Buenos Aires: UBA; 2006. p.1-25.

34. Guber R. La etnografía: método, campo y reflexividad. Buenos Aires: Norma; 2001.

35. Barómetro de la Deuda Social Argentina (BDSA). Estudio de la situación del desarrollo humano y social. Serie del Bicentenario (2010-2016)/Año 1. [Internet]. Buenos Aires: Observatorio de la Deuda Social Argentina. Universidad Católica Argentina; 2011 [acceso el 2014 Ago 19]. Disponible en: http://www.uca.edu.ar/uca/common/grupo68/ files/2011-08-01-informe-barometro-deuda-social-argentina.pdf

36. Kessler G, Svampa M, González Bombal I. Reconfiguraciones del mundo popular: el Conurbano Bonaerense en la postconvertibilidad. Buenos Aires: Prometeo-UNGS; 2010.

37. Svampa M. La sociedad excluyente: la Argentina bajo el signo del neoliberalismo. Buenos Aires: Taurus; 2005.

38. Van Zanten A. ¿El fin de la meritocracia? Cambios recientes en las relaciones de la escuela con el sistema económico, político y social. In: Tenti Fanfani E, compilador. Nuevos temas en la agenda de política educativa. Buenos Aires: Siglo XXI; 2008. p. 173-92.

39. Merklen D. Pobres ciudadanos: las clases populares en la era democrática Argentina (1983-2003). 2a ed. Buenos Aires: Gorla; 2010. 
40. Noel G. La conflictividad cotidiana en el escenario escolar: una perspectiva etnográfica. Buenos Aires: UNSAM; 2009.

41. Reguillo Cruz R. Instituciones desafiadas: subjetividades juveniles - territorios en reconfiguración. In: Tenti Fanfani E, compilador. Nuevos temas en la agenda de política educativa. Buenos Aires: Siglo XXI; 2008. p. 125-43.

42. Salvia A, Chávez Molina E. Sombras de una marginalidad fragmentada: aproximaciones a la metamorfosis de los sectores populares de la Argentina. Buenos Aires: Miño y Dávila; 2007.

43. Urresti M. De la cultura del aguante a la cultura del reviente: cambios en la significación de la corporalidad en adolescentes y jóvenes de sectores populares. In: Margulis $M$, Urresti $M$, Lewin $H$, compiladores. Familia, hábitat y sexualidad en Buenos Aires: investigaciones desde la dimensión cultural. Buenos Aires: Biblos; 2007. p. 281-92.

44. Kessler G. Redefinición del mundo social en tiempos de cambio: una tipología para la experiencia de empobrecimiento. In: Svampa $M$, editora. Desde abajo: la transformación de las identidades sociales. 2a ed. Buenos Aires: Biblos, Universidad Nacional de General Sarmiento; 2000. p. 25-50.

45. Gutierrez L, Romero L. A. Sectores populares, cultura y política. Buenos Aires: Sudamericana; 1995.

46. Weber M. La ética protestante. 2a ed. Buenos Aires: Libertador; 2007.

Wald G. Arte e Saúde: algumas considerações para aprofundar as potencialidades de análise do campo. Interface (Botucatu). 2015; 19(55):1051-62.

O artigo debate os princípios mais correntes do campo "arte e saúde", especificamente aqueles estudos de origem anglo-saxã que analisam programas de arte baseados na comunidade. Apresenta resultados de uma pesquisa qualitativa realizada em dois projetos de orquestras juvenis, da cidade de Buenos Aires, Argentina, visando à integração social. O trabalho denota como as possibilidades de influência de um projeto de educação artística dependem não só do que os programas oferecem, mas, também, fundamentalmente, do que os participantes trazem e são: os seus universos normativos e de crença, os seus esquemas de interpretação, posicionamentos morais, repertórios culturais, saberes práticos, memórias, trajetórias econômicas, trabalhistas e educativas. Estes aspectos, que são o resultado de processos prévios de socialização, constituem, de alguma forma, um filtro ou peneira através e a partir dos quais as intervenções artísticas podem - ou não - resultar apropriadas.

Palavras-chave: Orquestras juvenis. Arte e Saúde. Jovens. Vulnerabilidade. Educação artística. 